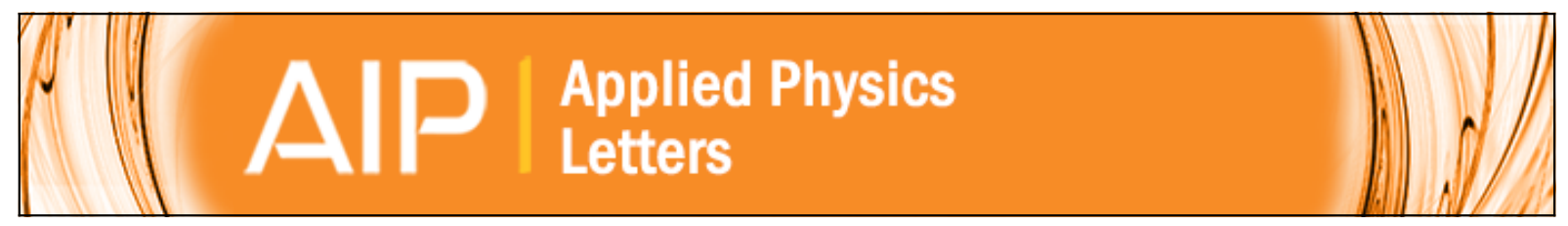

High frequency components of current fluctuations in semiconductor tunneling barriers

Xavier Oriols, Ferran Martn, and Jordi Suñé

Citation: Applied Physics Letters 80, 4048 (2002); doi: 10.1063/1.1482136

View online: http://dx.doi.org/10.1063/1.1482136

View Table of Contents: http://scitation.aip.org/content/aip/journal/apl/80/21?ver=pdfcov

Published by the AIP Publishing 


\title{
High frequency components of current fluctuations in semiconductor tunneling barriers
}

\author{
Xavier Oriols, ${ }^{\text {a) }}$ Ferran Martín, and Jordi Suñé \\ Departament d'Enginyeria Electrònica-ETSE, Universitat Autónoma de Barcelona, 08193-Bellaterra, Spain
}

(Received 7 November 2001; accepted for publication 29 March 2002)

\begin{abstract}
The power spectral density of current noise in phase-coherent semiconductor tunneling scenarios is studied in terms of Bohm trajectories associated to time-dependent wave packets. In particular, the influence of the particles reflected by the barrier on the noise spectrum is analyzed. An enhancement of the power spectral density of the current fluctuations is predicted for very high frequencies. The experimental measurement of this high frequency effect is discussed as a possible test of Bohm trajectories. (C) 2002 American Institute of Physics. [DOI: 10.1063/1.1482136]
\end{abstract}

The Landauer approach ${ }^{1}$ has become the standard method to treat steady-state coherent transport in mesoscopic systems. Using the second quantization framework, Lesovik ${ }^{2}$ and Buttiker ${ }^{3}$ extended the previous approach to study noise properties in phase-coherent systems. In particular, the power spectral density of current fluctuations at low frequencies for one-dimensional systems is expressed as

$$
\begin{aligned}
S(0)= & \frac{q^{2}}{\pi \hbar} \int_{0}^{\infty}\left\{T\left[f_{L} \cdot\left(1-f_{R}\right)+f_{R} \cdot\left(1-f_{L}\right)\right]\right. \\
& \left.-T^{2}\left(f_{L}-f_{R}\right)^{2}\right\} d E,
\end{aligned}
$$

where $q$ is the absolute value of the electron charge. The transmission coefficient, $T$, and the Fermi-Dirac occupation functions at the left (right) reservoir, $f_{L / R}$, depend on the electron energy $E$. Based on a wave packet formalism, Landauer and Martin ${ }^{4}$ provide a simple alternative derivation for $S(0)$. For each small energy interval, $\Delta E$, the current can be represented as a set of $\delta$ pulses (each one with an absolute area $q$ ) which account for the random transmission of electrons between the reservoirs. A positive pulse is measured when a wave packet incident from the left electrode is transmitted to an empty state in the right reservoir. The probability of this event is $T \cdot f_{L}\left(1-f_{R}\right)$, where the $1-f_{R}$ factor accounts for the Pauli principle. The transmission from right to left gives a negative pulse whose probability is $T \cdot f_{R}(1$ $-f_{L}$ ). For a single injection event, the average value of charge detected at the left contact is defined as $\bar{Q}=q \cdot T$ - $\left(f_{L}-f_{R}\right)$. The noise is computed as the variance $\overline{\Delta Q^{2}}$ $=\overline{Q^{2}}-(\bar{Q})^{2}$. The expression for $S(0)$ is obtained when $\overline{\Delta Q^{2}}$ is integrated over the whole energy range multiplied by the one-dimensional density of incoming electrons $5,6 \nu$ $=\Delta E / \hbar \pi$. In a recent letter, ${ }^{7}$ we have shown that this alternative picture for electronic noise is quite naturally supported within Bohm interpretation of quantum mechanics (QM). ${ }^{8}$ In this work, we investigate the role of reflected particles in the power spectral density of current fluctuations in semiconductor tunneling scenarios. At high frequencies, we predict an enhancement of the current noise spectrum due to

a)Electronic mail: xavier.oriols@uab.es reflected particles. These high frequency features are studied in detail in order to discuss its experimental measurement.

Since our approach ${ }^{7}$ follows Bohm formalism ${ }^{8}$ of QM that deals with well-defined trajectories, the current flowing through a mesoscopic electronic device has a clear meaning. Pellegrini, according to the extension of the Ramo-Shockley theorem to semiconductor, provides an expression to connect the electron motion inside the device to the external current. ${ }^{9}$ The total instantaneous current, $I(t)$, consisting of conduction and displacement current, is given by

$$
I(t)=\frac{q}{L} \sum_{i=1}^{N(t)} \nu_{i}(x, t),
$$

where $L$ is the length of the device (defined as the distance between the two electrodes which are maintained at constant potentials), $N(t)$ is the total number of carriers which are instantaneously inside the device, and $v_{i}(x, t)$ is the value of

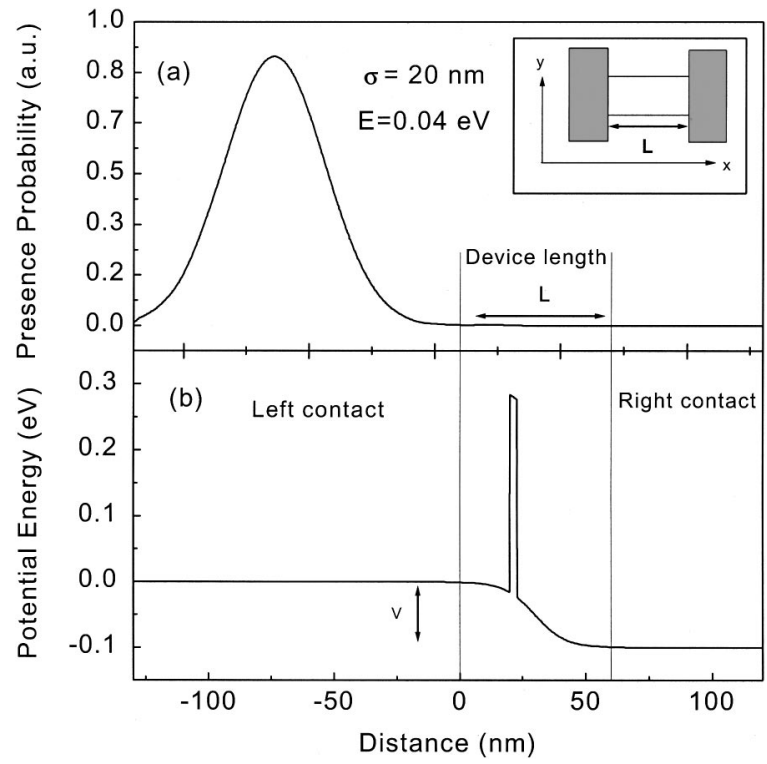

FIG. 1. Sketch of the simulating region that includes the device $(0<x$ $<L$ ) and the doped contacts. (a) Probability of the initial wave packet (the inset shows the lateral dimensions of the whole system); (b) potential energy profile along the contacts and the device region. 
the electron velocity at time $t$ and position $x$ (the level $i$ identifies each electron, and only those within $0<x<L$ have to be considered, see Fig. 1).

First, before working with the approach described in Ref. 7, let us discuss the influence of the transmitted and reflected particles on the power spectral density using a simplified analytical image. We consider a one-dimensional tunneling barrier where monoenergetic electrons are injected, without correlation between the initial times, from the left reservoir at an effective rate $\nu^{\prime}$. Assuming a constant velocity, $\nu_{0}$, for the transmitted electrons, a square pulse of height $q \cdot \nu_{o} / L$ and duration $\tau_{T}=L / \nu_{o}$ is expected in $I(t)$ for each transmitted electron. Identically to the Landauer image, ${ }^{10}$ the current obtained from Eq. (2) will be represented by positive and negative pulses each having an absolute area equal to $q$. Assuming low average current, $\Delta I(t)=I(t)-\bar{I} \approx I(t)$, the power spectral density of the current fluctuations can be computed by Fourier transforming the square-pulse autocorrelation. We obtain the well-known expression:

$$
S_{T}(x)=T \cdot \nu^{\prime} \cdot 2 \cdot q^{2} \sin ^{2}(x) / x^{2},
$$

where we defined $x=w \tau_{T} / 2$. At low frequency, we obtain a constant value $S_{T}(0)=2 \cdot q^{2} \cdot T \cdot \nu^{\prime}$ that decreases to zero at $w=2 \pi / \tau_{T}$. The Fano factor, $F$, defined as $S_{T}(0)=F \cdot 2 \cdot q \cdot \bar{I}$ takes the expected value $F=1$ since the initial assumption of low injection is equivalent to a Poissonian process.

In this letter, we investigate the role of the reflected electrons in the power spectral noise. We will show that these electrons do not contribute to $S(0)$ but provide an enhancement of $S(f)$ at high frequency components. All transmitted electrons have to cross the device. On the contrary, if the size of the electron wave packet is large in comparison with the device length (which is a common situation in mesoscopic systems), most of the electrons will be reflected inside the contacts. However, we argue that there are systems where the role of the reflected particles could modify the actual noise power spectral density. In this regard, we can expect that a reflected electron will be roughly monitored in $I(t)$ by a linear triangular pulse of initial height $q \cdot \nu_{o} / L$ and final height $-q \cdot \nu_{o} / L$ (i.e., the total area of the reflected electrons is zero since they do not contribute to the average current). The duration of the pulse, $\tau_{R}$, is not only related to the length of the device, but also to the wave-packet dynamics. Following the previous development, the power spectral density associated to reflected electrons is: ${ }^{11}$

$$
\begin{aligned}
S_{R}\left(x^{\prime}\right)= & (1-T) \cdot \nu^{\prime} \cdot \frac{2 \cdot q^{2} \cdot \tau_{R}^{2} \cdot v_{o}^{2}}{L^{2}} \\
& \cdot \frac{x^{\prime 2} \cdot \cos ^{2}\left(x^{\prime}\right)+\sin ^{2}\left(x^{\prime}\right)-x^{\prime} \cdot \sin \left(2 \cdot x^{\prime}\right)}{x^{\prime 4}},
\end{aligned}
$$

where $x^{\prime}=w \tau_{R} / 2$. At low frequencies, we obtain the value $S_{R}(0)=0$ and at $x^{\prime} \approx 2$ the maximum value $S_{R}(2) \approx 0.4 \cdot(1$ $-T) \cdot \nu^{\prime} \cdot q^{2} \cdot \nu_{o}^{2} \cdot \tau_{R}^{2} / L^{2}$. Thus, an enhancement of high frequency components of the total spectral power can be expected due to reflected particles.

In order to discuss the experimental measurement of this effect, we make a numerical simulation (using the approach described in Ref. 7) of the current noise for a single tunnel- ing barrier in the well-known GaAs/AlAs systems. A $0.3 \mathrm{eV}$ height and $3 \mathrm{~nm}$ length barrier of $\mathrm{Al}_{x} \mathrm{Ga}_{1-x}$ As is sandwiched between two intrinsic GaAs spacer layers of 20 and $37 \mathrm{~nm}$ for the left/right, respectively. Two additional doped GaAs layers with a wider lateral area [see inset of Fig. 1(a)] are characterized as perfect reservoirs (we assume that the lateral area of the device is large enough to validate the use of the one-dimensional approach). The voltage, $V=0.1 \mathrm{~V}$, is high enough to consider only injection from the left contact (we use $f_{L}=0.057$ at $300 \mathrm{~K}$ ). In order to compute Bohm trajectories, we numerically calculate the time evolution of a wave packet $\Psi(x, t)$ by solving the time-dependent Schrödinger equation in the whole simulation box that includes the device and the contacts. A simulating box of $2048 \AA$ (with a spatial step of $2 \AA$ ) and an electron effective mass equal to $m^{*}=0.067 m_{o}$ (being $m_{o}$ the free electron mass) is used. For simplicity, we considered wave packets with a unique kinetic energy equal to $E=0.04 \mathrm{eV}$ and positive central wave vector, $k_{c}$, computed from the parabolic $E-k$ relation. As seen in Fig. 1(a), an initial Gaussian wave packet of spatial dispersion $^{12} \sigma=20 \mathrm{~nm}$ located inside the left contact is used. According to Levitov and co-workers, ${ }^{13}$ electrons are injected into the sample following a binomial distribution. In particular, we use a particle Monte Carlo scheme developed by Gonzalez et al. ${ }^{14}$ At each time interval of duration, $\nu^{-1}$ $=m^{*} \pi / \hbar k_{c} \Delta k$ an attempt to introduce a wave packet takes place (in our case, $\Delta k$ is the inverse of the wave packet spatial dispersion $\Delta k=1 / \sigma$ as discussed in footnote 6 ). Then a random number $r$ uniformly distributed between zero and one is generated, and the attempt is considered successful only if $r<f_{L}$. Hence, the effective injection rate can be defined as $\nu^{\prime}=\nu \cdot f_{L}$. On the other hand, each time an electron definitively enters the simulation box, its initial position $x_{o}$ is randomly selected from the probability $\left|\Psi\left(x_{o}, t=0\right)\right|^{2}$ that takes into account the partition noise via the unavoidable uncertainty of QM. Once the $x_{o}$ is known, the causal trajectory is computed by integrating the instantaneous Bohm velocity. ${ }^{15,16}$ As depicted in Fig. 1(b), a constant potential profile (roughly similar to those obtained by self-consistently solving Schrödinger and Poisson equations) is assumed along the whole device region. A purely ballistic electron transport without scattering is considered.

In Fig. 2(a) we represent the current noise power of the system. The instantaneous current, $I(t)$ is obtained from Eq. (2) by simulating during $500 \mathrm{ps}$ (with a temporal step of 10 fs) random Bohm trajectories. The total power spectral density of the current fluctuations, $S(f)$, are obtained following the standard classical MC method. ${ }^{7,17}$ We have separately plotted the contribution to $S(f)$ due to transmitted and reflected electrons. From these results, we clearly show an enhancement of the power spectral density at the frequency range from 1 to $8 \mathrm{THz}$. Figure 2(b) also depicts the expressions for $S_{T}(f)$ and $S_{R}(f)$ obtained from the simple analytical model developed in the text. The average transmission coefficient of the wave packet is $T=0.08$. The device length is $L=600 \AA$. The transmission time, obtained from the numerical simulation, gives $\tau_{T} \approx 0.15 \mathrm{ps}$ and the reflection time is roughly $\tau_{R} \approx 0.12 \mathrm{ps}$. The velocity, obtained as an average value of the numerically simulated transmitted electrons, is $v_{o}=0.36 \times 10^{6} \mathrm{~m} / \mathrm{s}$. The effective injection rate is given by 


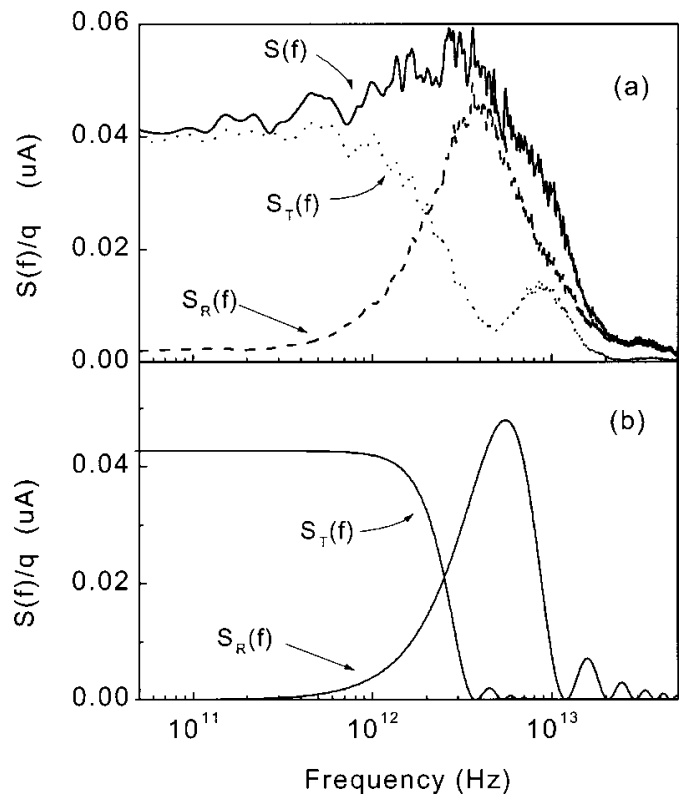

FIG. 2. One-side current noise power spectral density computed from numerical simulation (a), and from analytical expressions (b). The partial contributions from transmitted electrons, $S_{T}(f)$ and reflected electrons $S_{R}(f)$ are depicted. An enhancement of the total $S(f)$ in the range between 1 and $8 \mathrm{THz}$, due to reflected electrons, is clearly manifested.

$v^{\prime}=1.66 \times 10^{12} \mathrm{~Hz}$. The differences between Figs. 2(a) and 2(b) are due to the fact that Bohm trajectories do not have a constant speed since its velocity inside (or near) the barrier decreases. Apart from these second-order differences, the results shown in Fig. 2 clearly confirm that reflected electrons can provide an enhancement of the very high frequency components of $S(f)$. One can expect that the terahertz spectrum will soon be experimentally accessible for studying noise since electronic engineers are now actively developing prototypes for signal sources and detectors at these frequencies. ${ }^{18,19}$ In this regard, the measurement of these high frequencies features can be used to discern if Bohm trajectories provide a useful picture to understand electronic transport.

In conclusion, using our approach based on Bohm trajectories associated to time dependent wave packets, ${ }^{7}$ we predicted an enhancement of the current noise spectrum at very high frequencies in phase-coherent devices. Since we deal with time dependent wave packet, we are able to capture the internal dynamic time scales related with nonzero frequencies. An effort is made to discuss a possible device where such an effect can be observed. Its experimental measurement will provide excellent evidence of the ability of Bohm trajectories ${ }^{8}$ to describe the electronic transport in mesoscopic systems. On the other hand, the scheme described in this work, coupled with a Poisson solver, opens an interesting path to study the effects of long range Coulomb interaction in the finite-frequency noise characteristics of phasecoherent devices. Future work will follow this direction.
The authors are grateful to Emilio Méndez, Javier Mateos, Tomas González, and Daniel Pardo for helpful discussion. This work has been partially supported by the Dirección General de Enseñanza Superior e Investigación through Project No. BFM2000-0353 and by a grant of the Ministerio de Educación Cultura y Deporte PR2001-0417.

${ }^{1}$ R. Landauer, IBM J. Res. Dev. 1, 223 (1957).

${ }^{2}$ G. B. Lesovik, JETP Lett. 49, 592 (1989); see also B. Yurke and G. P. Kochanski, Phys. Rev. B 41, 8184 (1990).

${ }^{3}$ M. Büttiker, Phys. Rev. Lett. 65, 2901 (1990).

${ }^{4}$ R. Landauer and Th. Martin, Physica B 182, 288 (1992); Th. Martin and R. Landauer, Phys. Rev. B 45, 1742 (1992).

${ }^{5}$ Y. Imry, Introduction to Mesoscopic Physics (Oxford University Press, London, 1997).

${ }^{6}$ One-dimensional density of incoming electrons can also be deduced form a QM wave-packet approach by analyzing the possibility of injecting two spatially overlapping wave packets. The antisymmetrical expression of two identical Gaussian wave packet (with identical central momentum $k_{c}$, spatial dispersion $\sigma$, but located at different central positions separated by a distance $d)$ is described by $\Psi\left(x_{1}, x_{2}, t\right)=1 / \sqrt{2}\left[\Psi_{1}\left(x_{1}, t\right) \cdot \Psi_{2}\left(x_{2}, t\right)\right.$ $\left.-\Psi_{1}\left(x_{2}, t\right) \cdot \Psi_{2}\left(x_{1}, t\right)\right]$. The total probability of finding the two electrons can be computed as $\iint\left|\Psi\left(x_{1}, x_{2}, t\right)\right|^{2} \cdot d x_{1} d x_{2}=1-\exp \left[-d^{2} /(2 \sigma)\right]$. This equation means that two identical wave packets initially closer than $d$ have a total probability near zero. In other words, wave packets have to be introduced into the sample at an injection rate equal or larger than approximately $v_{c} / \sigma$, where $v_{c}$ is the central velocity of the initial Gaussian wave packet. In the parabolic band approximation we find $\nu_{c}=\hbar k_{c} / m^{*}$ and $1 / \sigma=\Delta k=m^{*} \cdot \Delta E /\left(\hbar^{2} k_{c}\right)$. The final expression for the density of incoming electrons, $\nu=\Delta E / \hbar \pi$, is reproduced by multiplying both expressions and taking into account the density in $k$ space, $1 / 2 \pi$, and a factor 2 for the spin. A similar demonstration, within the language of the second quantization applied to wave packets, can be found on page 16 of reference; Ya. M. Blanter and M. Buttiker, Phys. Rep. 336, 1 (2000).

${ }^{7}$ X. Oriols, F. Martín, and J. Suñé, Appl. Phys. Lett. 79, 806 (2001).

${ }^{8}$ D. Bohm, Phys. Rev. 85, 166 (1952).

${ }^{9}$ B. Pellegrini, Phys. Rev. B 34, 5921 (1986); G. Cavalleri, E. Gatti, G. Fabbri, and V. Svelto, Nucl. Instrum. Methods 92, 137 (1971).

${ }^{10}$ R. Landauer, Physica D 38, 226 (1989).

${ }^{11}$ Reflected particles are represented in the current by a sawtooth antisymmetric pulse. The autocorrelation function, $C_{R}(t)$, of this current pulse is described by $C_{R}(t)=(1-T) \cdot \nu^{\prime} \cdot q^{2} \cdot \nu_{o}\left(\tau_{R}^{3}-3 \tau_{R}^{2}|t|+2|t|^{3}\right) /\left(3 \tau_{R}^{2} L^{2}\right)$ for $|t| \leqslant \tau_{R}$ and $C_{R}(t)=0$ elsewhere. The one-side power spectral density is computed by Fourier transforming the previous autocorrelation function and multiplying by 2 .

${ }^{12}$ Although there is not formal proof, a reasonable value of the size of wave-packet length is given as $\sim 50 \mathrm{~nm}$ on p. 276: M. V. Fischetti, J. Appl. Phys. 83, 270 (1998); The size of the electron considered in our work can be estimated as $2 \sigma=40 \mathrm{~nm}$.

${ }^{13}$ L. S. Levitov and G. B. Lesovik, JETP Lett. 58, 230 (1993); L. S. Levitov, H. Lee, and G. B. Lesovik, J. Math. Phys. 37, 4845 (1996).

${ }^{14}$ T. González, J. Mateos, D. Pardo, L. Varani, and L. Reggiani, Semicond. Sci. Technol. 14, L37 (1999).

${ }^{15}$ X. Oriols, J. J. García, F. Martín, J. Suñé, T. Gonzalez, J. Mateos, and D. Pardo, Appl. Phys. Lett. 72, 806 (1998).

${ }^{16}$ X. Oriols, J. J. Garcia, F. Martín, J. Suñé, T. González, J. Mateos, D. Pardo, and O. Vanbesien, Semicond. Sci. Technol. 14, 532 (1999).

${ }^{17}$ L. Varani, L. Reggiani, T. Kuhn, T. González, and D. Pardo, IEEE Trans. Electron Devices 41, 1916 (1994).

${ }^{18}$ M. J. W. Rodwell, S. T. Allen, R. Y. Yu, M. G. Case, U. Bhattacharya, M. Reddy, E. Carman, M. Kamegawa, Y. Konishi, J. Pusl, and R. Pulella, Proc. IEEE 82, 1037 (1994).

${ }^{19}$ M. Reznikov, M. Heiblum, H. Shtrikman, and D. Mahalu, Phys. Rev. Lett. 75, $3340(1995)$. 